
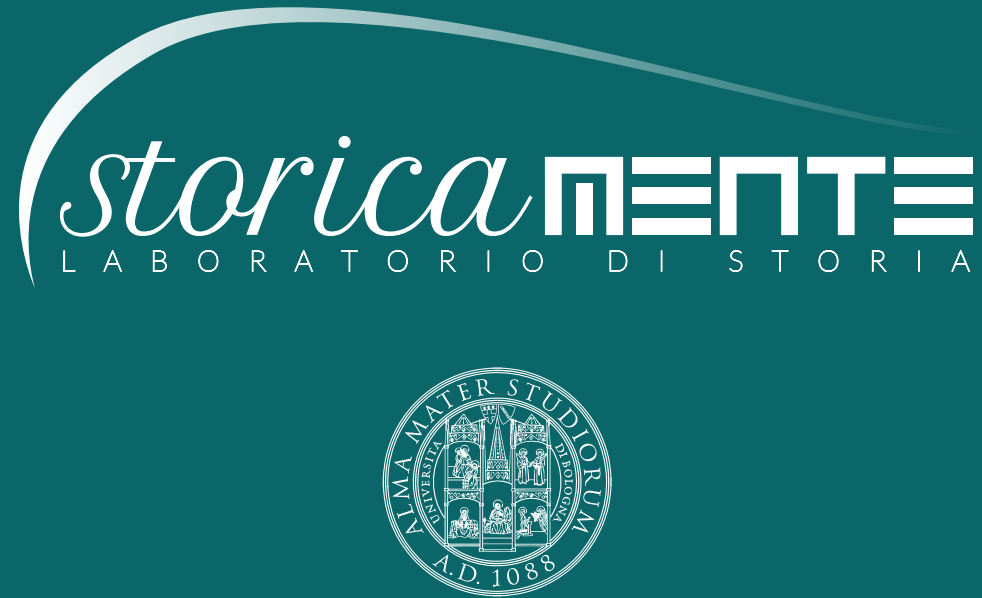

ALMA MATER STUDIORUM

Università di Bologna

Dipartimento di Storia Culture Civiltà

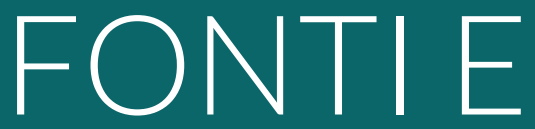




\section{STORICAMENTE.ORG}

\section{Laboratorio di Storia}

Luca Tedesco

La sicurezza nelle miniere sarde tra Otto e Novecento: cosa ci raccontano le statistiche

Numero $14-2018$

ISSN: $1825-411 \mathrm{X}$

Art. 32

pp. $1-11$

DOI: $10.12977 /$ stor722

Editore: BraDypUS

Data di pubblicazione: 11/01/2019

Fonti e documenti: Frammenti di storia del lavoro nelle miniere sarde: tra archivi e memoria, a cura di Luca Tedesco 


\title{
La sicurezza nelle miniere sarde tra Otto e Novecento: cosa ci raccontano le statistiche
}

\author{
LUCA TEDESCO
}

Univ. Roma Tre, Dipartimento di Scienze della Formazione

The interpretation provided by the historiography in the field of security in Sardinian mines in the Italy of the second industrial revolution is darkened, an interpretation that makes the mine a very insecure place, marked by a dripping of tragedies and fatal accidents. Nevertheless, the above reading does not appear to be fully justified by the available statistical data which, on the contrary, allows us to advance the thesis that, starting from the beginning of the 1980s up until 1910, the number of deaths by accident in the Sardinian mines in relation to the mining population has been below the national average .

\section{La sicurezza nelle miniere sarde tra Otto e Novecento}

La lettura fornita dalla storiografia in materia di sicurezza nelle miniere sarde nell'Italia della seconda rivoluzione industriale è una lettura incupita, a tinte fosche, che fa della miniera un luogo fortemente insicuro, segnato da uno stillicidio di tragedie e infortuni mortali. Questa lettura risulta infatti sostanzialmente condizionata dal tristemente noto incidente del 4 maggio 1871 nella miniera di Montevecchio, nella provincia del Sud Sardegna, che comprende dal 2016 sostanzialmente le ex province di Carbonia-Iglesias e Medio Campidano, incidente che 
avrebbe provocato la morte di undici donne, di cui otto non ancora quindicenni [Peis Concas 2006, 41-66].

Roberto Porrà, nel suo pregevole lavoro Infortuni sul lavoro nelle miniere sarde (1861-1886), ricorda poi la "serie agghiaccianti di numerosi - circa 90 - e impressionanti incidenti sul lavoro quasi tutti mortali, verificatisi dal 1861 al 1886, dei quali furono vittime lavoratori, spesso di giovane età, per lo più delle miniere dell'Iglesiente e del GuspineseArborese» [Porrà 1993, 70].

È stato d'altronde da più fonti sottolineato come la legislazione sulla sicurezza del lavoro e, più in generale, quella sociale, peraltro assai contenuta fino alla fine del XIX secolo [Manconi 1986, 72]', abbia trovato in Sardegna un'attuazione spesso lacunosa [Manconi 1986, 71-2; Porrà 1993, 70-71; Todde 2013, 297-298].

Ciononostante, la lettura di cui sopra non appare a nostro avviso pienamente giustificata dai dati statistici disponibili che permettono anzi di avanzare l'ipotesi che, a partire perlomeno dall'inizio degli anni Ottanta e fino alla fine del periodo considerato, il 1910, anno di pubblicazione degli Atti della Commissione parlamentare d'inchiesta sulla condizione degli operai delle miniere della Sardegna, il numero dei morti per incidente nelle miniere sarde in rapporto alla popolazione mineraria (per ragioni

Ci riferiamo alla legge del 20 novembre 1859, applicata in Piemonte, Sardegna e Lombardia, concernente la tutela degli infortuni e la sicurezza del lavoro, al regio decreto 23 dicembre 1865, sempre in tema di sicurezza sul luogo di lavoro, alla legge dell's luglio 1883 istituente la Cassa Nazionale di Assicurazione contro gli Infortuni sul Lavoro, cassa, peraltro a contribuzione volontaria e quindi pressoché ignorata dai minatori sardi, alla legge dell'11 febbraio 1886 sul lavoro dei fanciulli nelle fabbriche, cave e miniere che fissava limiti su età e orario di lavoro, alla legge 30 marzo 1893 sulla polizia delle miniere, cave e torbiere, alla legge del 15 giugno 1893 che creava il Collegio dei Probiviri per le controversie di lavoro, alla legge del 17 marzo 1898 sugli infortuni di lavoro, a quella del 17 luglio dello stesso anno istituente una Cassa di Previdenza per le Malattie e la Vecchiaia e al regio decreto del 18 giugno 1899, recante il regolamento per la prevenzione degli infortuni nelle imprese e nelle industrie che trattavano materiali esplosivi. 
la cui investigazione esula da questo saggio) sia stato al di sotto della media nazionale.

Già la letteratura coeva, non sufficientemente valorizzata dalla storiografia, aveva in verità rilevato tale fenomeno.

Il medico Gildo Frongia, specialista delle condizioni igieniche e sanitarie nel mondo estrattivo [Ruju 1999, 328], in base ai dati offerti dal Sindacato per gli Infortuni sul Lavoro nelle Coltivazioni di Miniera relativamente al periodo gennaio 1898-dicembre 1909 scriveva come in Sardegna il numero degli infortunati fosse «diminuito sensibilmente dopo le applicazioni delle leggi di polizia mineraria, e specialmente negli ultimi anni in cui si è resa sempre più attenta la sorveglianza tecnica, e si sono adottate efficaci norme preventive di sicurezza con l'aumentare delle difficoltà o pericoli dei lavori» [Frongia 1911, 126-127].

Marcello Vinelli definiva le condizioni di sicurezza dell'industria mineraria sarda "ottime» [Vinelli 1914, 65], facendo riferimento alle risultanze della citata commissione parlamentare d'inchiesta.

Questa, in verità, le aveva definite addirittura «eccellenti», sia sotto il profilo della graduale diminuzione dei casi di morte (per mille operai, 1,01 nel periodo $1874-1879 ; 2,00$ nel periodo $1880-1889 ; 1,18$ nel periodo 1890-1899; 0,74 nel periodo 1900-1907) sia sotto il profilo della comparazione dell'ultimo coefficiente medio (relativo all'intervallo 1900-1907) con quello delle miniere degli altri Paesi, quale era desumibile dai dati prodotti dall'economista e geologo Louis de Launay [1903] e dall'«Engineering and Mining Journal» di New York [1910], attestanti una mortalità superiore in Austria-Ungheria, in Francia, in Spagna, in Gran Bretagna e negli Stati Uniti [Commissione 1910, II, 19].

Ciò era particolarmente significativo se si teneva a mente che la netta flessione del periodo 1900-1907 coincideva con la diminuzione della ricchezza dei giacimenti e il conseguente incremento dell'impiego della forza motrice.

Del resto - continuava la relazione - la Commissione ha potuto formarsi il concetto della regolarità tecnica, della perfetta organizzazione, della 
capacità dei dirigenti che distinguono l'industria mineraria di Sardegna e che le costituiscono massimo titolo d'onore. Né a questo risultato è estranea l'opera dell'ufficio minerario di Iglesias, sia per la sorveglianza esercitata, sia per l'andamento impresso alla scuola mineraria che ha fornito e fornisce valente personale tecnico [Commissione 1911, I, 71].

La commissione precisava che l'industria mineraria dell'isola, a partire dagli anni Settanta dell'Ottocento, trovò in Sardegna «un ambiente pressoché vergine ed esente da preconcetti o da consuetudini locali», di modo che poté attuarsi sotto "la guida di tecnici esperti», seguendo «criteri razionali» e imponendo alla classe operaia "quella rigorosa disciplina di lavoro che è uno dei principali fattori della sicurezza delle coltivazioni» [Commissione 1910, II, 17]. Secondo la commissione, la sicurezza era rafforzata dal fatto che le miniere erano sfruttate da concessionari, interessati a "uno sfruttamento metodico dei giacimenti»; ciò che non avveniva in caso di affitti a breve scadenza che davano generalmente luogo a «lavorazioni disordinate» [Commissione 1910, II, 17]. Salvatorico Serra, però, nel suo denso saggio sul movimento sindacale nelle zone minerarie sarde, utilizza proprio i risultati statistici elaborati dal Sindacato per gli infortuni e riportati da Frongia per annotare che, oltre al malattie, anche "gli infortuni imperversavano nelle miniere sarde» (il tasso di mortalità per infortuni, peraltro, che Serra ricava dagli studi di una delle massime autorità dell'epoca in tema di malattie professionali dei minatori, il medico Alfonso Giordano [Giordano 1913, 187], si riferisce non alle miniere sarde ma alle solfatare siciliane) [Serra 2006, 95].

Eppure i dati prodotti da Puddu e Locci [Puddu, Locci 2006, 187292], frutto di un lavoro di scandaglio presso il Distretto minerario di Iglesias, i registri parrocchiali, gli Uffici anagrafici comunali e il Fondo sugli incidenti in miniera dell'Archivio di Stato di Cagliari, e pubblicati nello stesso volume collettaneo in cui è apparso il lavoro di Serra, sembrano confermare, nonostante difformità nei valori assoluti, quanto emerge da quelli ufficiali, apparsi sugli «Annali del Ministero di agricoltura, industria e commercio». Questa pubblicazione, nata nel 1870 
e divenuta nel 1891 la "Rivista del Servizio minerario», rappresenta il primo periodico di divulgazione economica, tecnica e scientifica in tema di miniere, ricco di dati statistici su produzione, ricerche minerarie, scoperte, infortuni nelle miniere e nelle cave, applicazione delle leggi riguardanti il lavoro e l'industria, espropriazioni, misure sanitarie, assistenziali e previdenziali: ciò grazie anche alla riproduzione delle relazioni degli Ispettori dei Distretti minerari.

Nella Prefazione al numero della "Rivista del Servizio minerario» relativo al 1891 si legge come a partire dal 1877 le relazioni inizino a riportare le statistiche della produzione mineraria [Pellati 1893, IX].

Per quanto riguarda le serie statistiche degli infortuni, la relazione del 1879 ammetteva la loro inattendibilità negli anni precedenti a causa della scarsa propensione degli esercenti l'attività estrattiva a denunciare gli infortuni stessi, atteggiamento che stava però mutando grazie alle "sollecitazioni degli uffici minerari» [Relazione sul servizio minerario nel 1879 1882, 29].

A partire dagli anni Ottanta, così, sarebbero apparse sistematicamente nei numeri della «Rivista del Servizio minerario» le statistiche relative agli infortuni nelle miniere, come si evince nella tabella riportata in calce. In essa abbiamo riportato tra parentesi tonde i dati relativi agli incidenti mortali (in valore assoluto e in percentuale) ricavati dal citato lavoro di Puddu e Locci. Per rendere confrontabili questi dati con quelli ufficiali (che non sono indicati tra parentesi), abbiamo conteggiato nel numero dei morti segnalati nella colonna "Distretto di Iglesias. Operai morti" (il Distretto minerario di Iglesias aveva la competenza per tutte le miniere isolane) anche i feriti deceduti nei giorni immediatamente successivi all'incidente (lo studio di Puddu e Locci prende infatti in considerazione anche i morti in ospedale).

Ciò che allora si evince da tale comparazione è che nel trentennio 1880-1910, anche assumendo come più attendibili le statistiche riprodotte nel lavoro di Puddu e Locci e che stimano un numero di operai morti maggiore rispetto ai dati ufficiali, la percentuale degli incidenti 
mortali in Sardegna risulta inferiore alla media nazionale, tranne che per gli anni 1884, 1885, 1891, 1892, 1893, 1896 e 1898 (non ci è stato possibile reperire i dati per il 1890). Tale minore incidenza dovrebbe essere confermata dall'ipotesi, ovviamente da sottoporre a verifica ma prima facie non infondata, che la sottostima del numero effettivo dei morti da parte delle statistiche ufficiali non abbia potuto riguardare solo il Distretto di Iglesias.

Da questa constatazione, peraltro, sarebbe scorretto inferire necessariamente una minore pericolosità complessiva della miniera sarda rispetto a quelle delle altre regioni italiane.

Lo stesso Frongia, infatti, contestando la relazione sanitaria contenuta negli atti della commissione, tacciata di minimizzare i rischi per la salute dell'ambiente minerario sardo [Ruju 1999, 328], rilevava come l'anemia che colpiva i minatori sardi fosse di natura tossica e non parassitaria come invece avveniva nelle altre regioni italiane e all'estero [Frongia 1911, 97]; altri segnalavano poi una maggiore incidenza tra i primi del saturnismo [Ruju 1999, 330]. Più in generale, accertata era la gravità nelle miniere sarde di malattie come la pneumoconiosi, la tubercolosi e la malaria [Ruju 1999, 330]. 


\begin{tabular}{|c|c|c|c|c|c|c|c|c|}
\hline 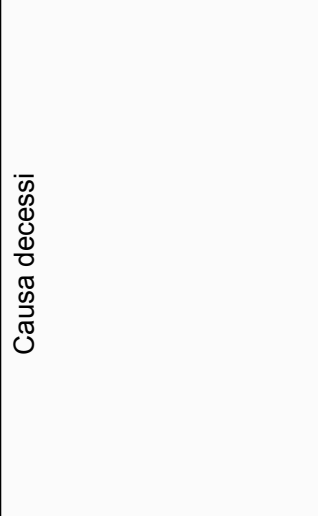 & 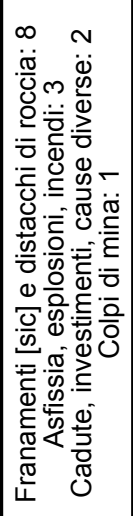 & 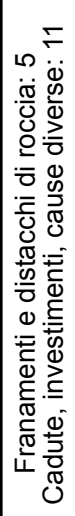 & 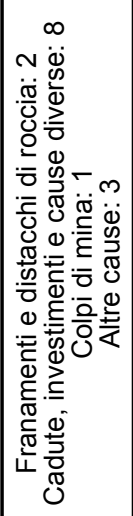 & 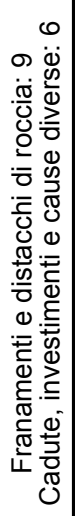 & 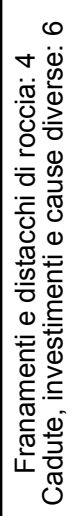 & 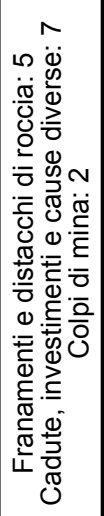 & 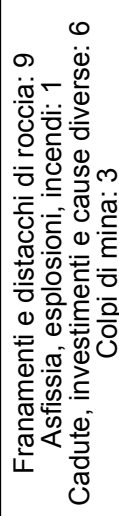 & 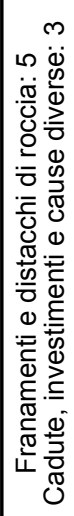 \\
\hline 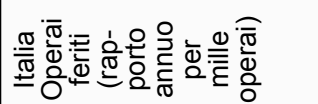 & $\stackrel{f}{-}$ & i & \begin{tabular}{l}
\multirow{2}{*}{} \\
m
\end{tabular} & 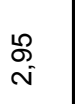 & S) & $\bar{\infty}_{\substack{\infty \\
N}}$ & $\begin{array}{l}\infty \\
0 \\
0 \\
0\end{array}$ & $\begin{array}{l}\sigma \\
\sigma \\
\sigma\end{array}$ \\
\hline 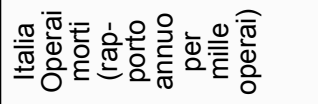 & $\frac{9}{i}$ & 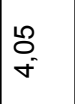 & în & 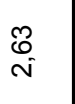 & 罟 & $\stackrel{\infty}{\infty}$ & 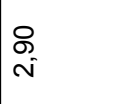 & m \\
\hline 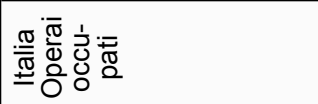 & 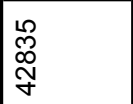 & 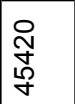 & 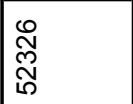 & 象 & 总 & 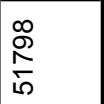 & 奂 & $\begin{array}{l}\mathscr{P} \\
0 \\
\& \\
\forall\end{array}$ \\
\hline 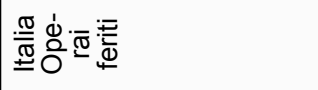 & $\mathscr{6}$ & $\stackrel{\mathbb{N}}{\underline{N}}$ & $\stackrel{9}{2}$ & 足 & 官 & 卓 & $\frac{m}{m}$ & 䒜 \\
\hline 霆 & ১ & $\underset{ \pm}{ \pm}$ & $\stackrel{\mathbb{N}}{\stackrel{4}{ }}$ & $\stackrel{\infty}{\stackrel{m}{?}}$ & $\infty$ & i & $\stackrel{m}{\square}$ & G \\
\hline 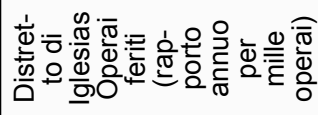 & $m_{\infty}^{\infty}$ & 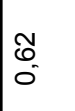 & 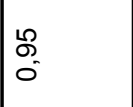 & $\stackrel{l}{0}_{0}$ & $8_{0}$ & 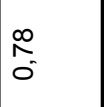 & $\underset{N}{\bar{N}}$ & $\begin{array}{l}2 \\
0 \\
0\end{array}$ \\
\hline 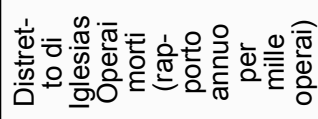 & 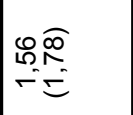 & 宓 & 奠 & 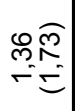 & $\stackrel{\substack{\infty \\
\check{c}}}{=}$ & 石 & 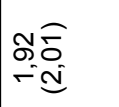 & 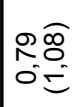 \\
\hline 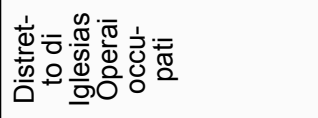 & $\begin{array}{l}\hat{A} \\
\mathscr{D} \\
\infty\end{array}$ & 紫 & |lo & 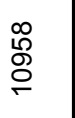 & $\begin{array}{l}\stackrel{2}{\circ} \\
\infty \\
\infty\end{array}$ & ્ָָ & $\frac{2}{4}$ & 商 \\
\hline 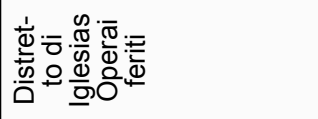 & $m$ & 0 & |으 & ما & 0 & $\infty$ & $\stackrel{2}{\simeq}$ & $\infty$ \\
\hline 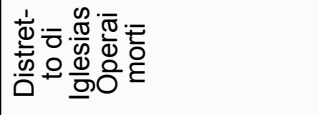 & $\forall \widehat{\theta}$ & $\stackrel{\circ \sigma}{=}$ & $\forall \stackrel{0}{=}$ & $\stackrel{2}{\circ}$ & 응 & 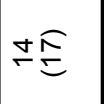 & 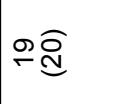 & $\infty \widehat{E}$ \\
\hline 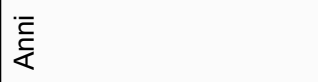 & $\begin{array}{l}\stackrel{\infty}{\infty} \\
\stackrel{\infty}{\sim}\end{array}$ & \begin{tabular}{|l}
$\bar{\infty}$ \\
$\stackrel{\infty}{\infty}$ \\
$\stackrel{\infty}{-}$
\end{tabular} & 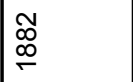 & 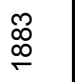 & $\begin{array}{l}\text { 总 } \\
\stackrel{\infty}{-}\end{array}$ & 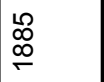 & $\begin{array}{l}\mathscr{\infty} \\
\stackrel{\infty}{\infty} \\
\stackrel{\infty}{-}\end{array}$ & $\begin{array}{l}\widehat{1} \\
\infty \\
\infty \\
-\infty\end{array}$ \\
\hline
\end{tabular}




\begin{tabular}{|c|c|c|c|c|c|c|c|c|c|}
\hline 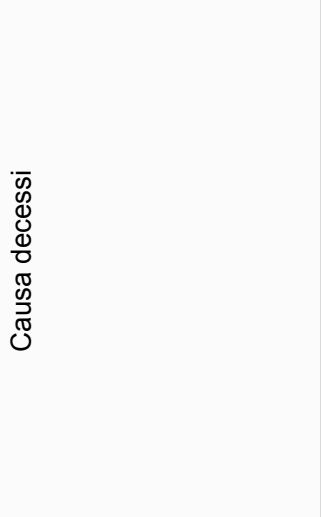 & 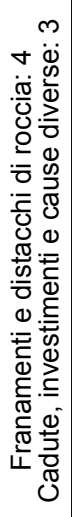 & 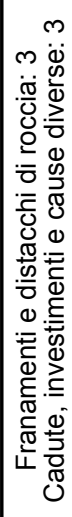 & & 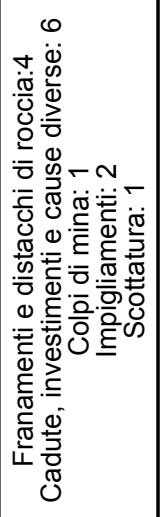 & 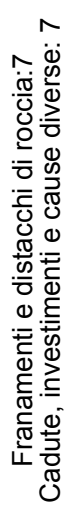 & 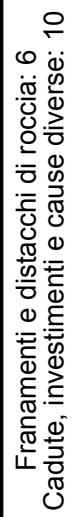 & 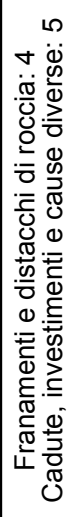 & 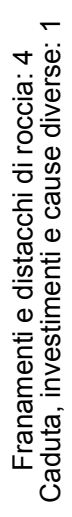 & 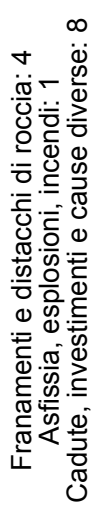 \\
\hline 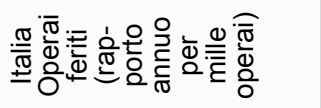 & $\begin{array}{l}\widetilde{N} \\
\infty \\
\infty\end{array}$ & 奋 & & $\stackrel{\substack{+\sim}}{i}$ & ले & 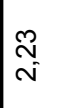 & $\frac{n}{m}$ & $\begin{array}{l}\infty \\
\infty \\
N\end{array}$ & 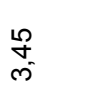 \\
\hline 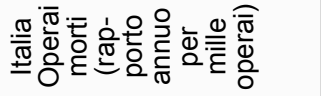 & $\stackrel{+}{\stackrel{N}{r}}$ & $\stackrel{m}{r}$ & & $\stackrel{8}{\circ}$ & $\underset{\sim}{\check{z}}$ & $\stackrel{m}{m}$ & 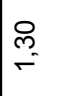 & $\stackrel{8}{\circ}$ & $\stackrel{\sigma}{\sigma}$ \\
\hline 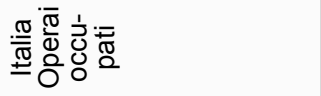 & 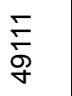 & $\begin{array}{l}-\bar{c} \\
\infty \\
\infty \\
\dot{\gamma}\end{array}$ & $\frac{8}{\text { กิ }}$ & 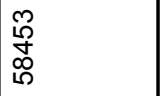 & 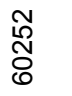 & 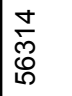 & $\frac{\hat{\Omega}}{\mathrm{S}}$ & 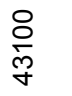 & $\begin{array}{l}\text { Ñ } \\
\text { लె } \\
\text { G }\end{array}$ \\
\hline 焉 & $\stackrel{\circ}{\sim}$ & $\stackrel{\Sigma}{\Sigma}$ & & 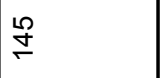 & $\check{\ddagger}$ & $\stackrel{\leftrightarrow}{\stackrel{N}{\sim}}$ & $\stackrel{+}{0}$ & $\stackrel{\stackrel{N}{N}}{\sim}$ & $\stackrel{8}{\circ}$ \\
\hline 疍 & $\widehat{\infty}$ & \begin{tabular}{|l|}
$\infty$ \\
0 \\
0
\end{tabular} & & రి & 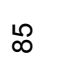 & $\stackrel{2}{R}$ & $\begin{array}{l}\infty \\
0\end{array}$ & $\overparen{0}$ & $\stackrel{R}{\sim}$ \\
\hline 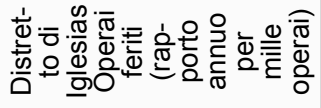 & $\begin{array}{l}\mathscr{8} \\
0 \\
0\end{array}$ & 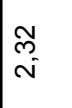 & & बू & $\stackrel{\leftrightarrow}{m}$ & $\begin{array}{l}\frac{10}{2} \\
0 \\
0\end{array}$ & $\stackrel{\infty}{\infty}$ & 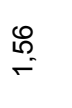 & $\begin{array}{l}\text { in } \\
\text { Ni }\end{array}$ \\
\hline 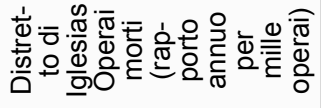 & $\begin{array}{l}\hat{6} \overline{\mathscr{O}} \\
0 \overline{0}\end{array}$ & $\begin{array}{l}\infty \\
\infty \\
0 \\
0 \\
0=\end{array}$ & & $\begin{array}{l}\overline{0} \\
\overline{0} \\
0=\end{array}$ & $\begin{array}{l}0 \widehat{N} \\
00 \\
0= \\
0=\end{array}$ & $\stackrel{5}{\circ}$ & 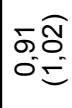 & $\begin{array}{l}1 \\
\text { గ్ } \\
0\end{array}$ & 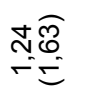 \\
\hline 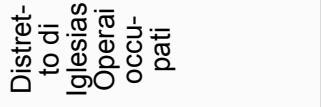 & 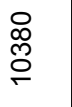 & 율 & & 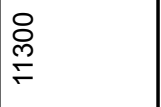 & $\underset{\substack{\infty \\
\leftarrow}}{\stackrel{0}{\leftarrow}}$ & 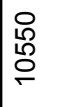 & $\begin{array}{l}8 \\
\circ \\
\circ\end{array}$ & 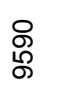 & 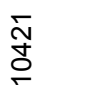 \\
\hline 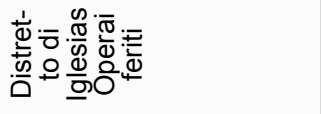 & 우 & d & & $\stackrel{20}{2}$ & $\infty$ & $\infty$ & $\stackrel{\infty}{\sim}$ & $\stackrel{2}{\leftarrow}$ & $\hat{\sim}$ \\
\hline 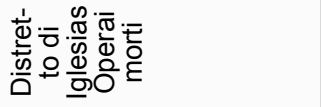 & $\wedge \stackrel{\circ}{=}$ & OE & $\underline{E}$ & 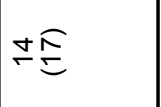 & $\stackrel{-\infty}{=}$ & $\ddot{0}$ & の읃 & $10 \widehat{\mathbb{J}}$ & $\stackrel{m}{\mathscr{F}}=$ \\
\hline 胥 & $\begin{array}{l}\infty \\
\infty \\
\infty \\
\leftarrow\end{array}$ & $\begin{array}{l}\mathscr{D} \\
\infty \\
\infty\end{array}$ & 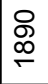 & ळ్ & ๙ু & ஜூ & 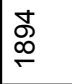 & 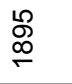 & $\begin{array}{l}\mathscr{0} \\
\infty \\
\infty\end{array}$ \\
\hline
\end{tabular}




\begin{tabular}{|c|c|c|c|c|c|c|c|c|}
\hline 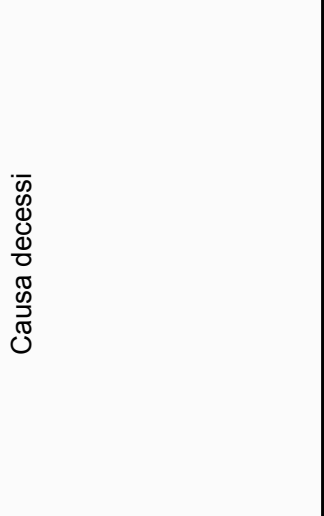 & 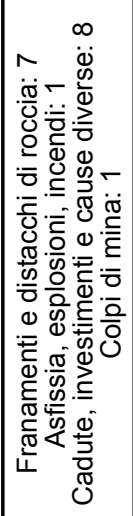 & 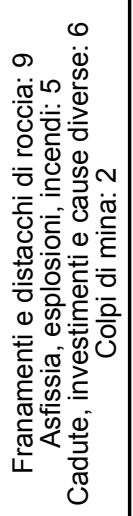 & 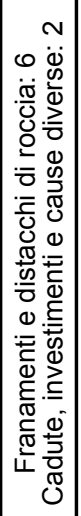 & 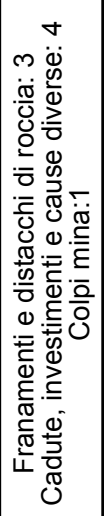 & 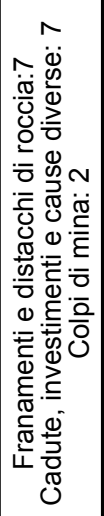 & 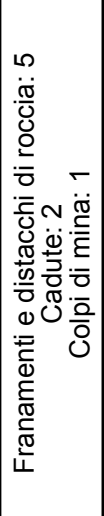 & & 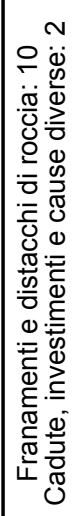 \\
\hline 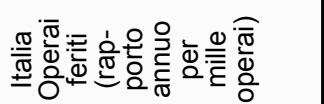 & $\begin{array}{l}\otimes \\
\vdots \\
i\end{array}$ & 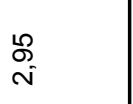 & $\underset{N}{N}$ & $\stackrel{\text { Lof }}{\text { s. }}$ & $\stackrel{R}{\stackrel{R}{N}}$ & 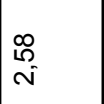 & 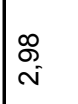 & \begin{tabular}{l}
0 \\
\multirow{i}{*}{}
\end{tabular} \\
\hline 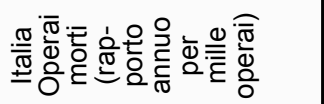 & 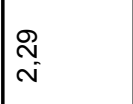 & $\underset{\leftarrow}{-}$ & $\stackrel{8}{-}$ & 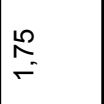 & 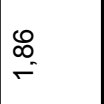 & $\stackrel{\leftrightarrow}{\stackrel{m}{r}}$ & 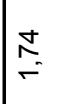 & $\underset{\sim}{\stackrel{\alpha}{\sigma}}$ \\
\hline 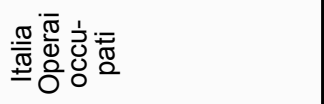 & 点 & $\begin{array}{l}9 \\
\substack{0 \\
\infty \\
i}\end{array}$ & 离 & 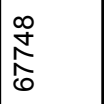 & $\begin{array}{l}0 \\
0 \\
0 \\
6\end{array}$ & $\begin{array}{l}\stackrel{0}{N} \\
\text { ల్ర }\end{array}$ & 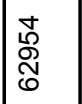 & ס̊ \\
\hline 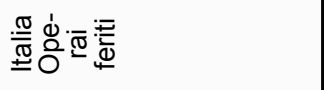 & 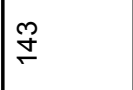 & $\nwarrow$ & 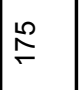 & $\stackrel{\circ}{\circ}$ & $\stackrel{\infty}{\infty}$ & ஜே & $\begin{array}{l}\infty \\
\stackrel{\infty}{\leftarrow}\end{array}$ & $\stackrel{\circ}{\circ}$ \\
\hline 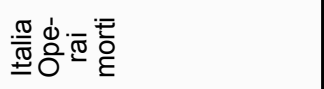 & $\stackrel{\stackrel{m}{N}}{\sim}$ & $\underset{\infty}{\infty}$ & 응 & $\stackrel{?}{\rightleftharpoons}$ & $\stackrel{\mathscr{N}}{\stackrel{N}{ }}$ & $œ$ & 읃 & 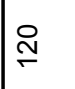 \\
\hline 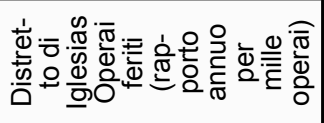 & 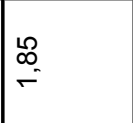 & 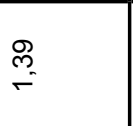 & $\begin{array}{l}\infty \\
\infty \\
0\end{array}$ & 只 & $\stackrel{\leftrightarrow}{m}$ & $\stackrel{\Xi}{\check{Z}}$ & $\stackrel{\text { g }}{\sim}$ & $\stackrel{\underset{\sim}{\sim}}{\sim}$ \\
\hline 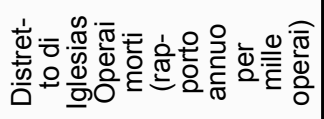 & $\stackrel{8}{\circ}$ & 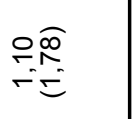 & 艿 & 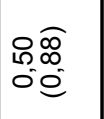 & $\underset{\substack{0 \\
\hdashline}}{-=}$ & $\begin{array}{l}-\widehat{\Omega} \\
0.0 \\
00\end{array}$ & 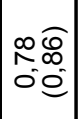 & $\mid \begin{array}{cc}\widehat{c} \\
\infty \\
\infty \\
0 \\
0=-\infty\end{array}$ \\
\hline 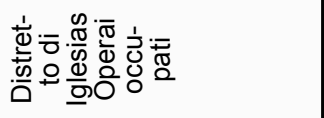 & $\stackrel{\circ}{\stackrel{0}{m}}$ & $\stackrel{\mathscr{D}}{\stackrel{\mathscr{D}}{\sim}}$ & 令 & 宮 & 薄 & 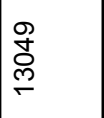 & $\begin{array}{l}\stackrel{0}{2} \\
\stackrel{\leftrightarrow}{N} \\
\stackrel{2}{\leftarrow}\end{array}$ & $\begin{array}{l}\infty \\
\infty \\
0 \\
\stackrel{0}{\longrightarrow} \\
\end{array}$ \\
\hline 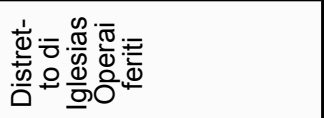 & $\bar{N}$ & $\stackrel{\infty}{\sim}$ & $\cong$ & $\stackrel{\sim}{\sim}$ & $\bar{N}$ & $\stackrel{\circ}{\leftarrow}$ & $\stackrel{g}{\leftarrow}$ & $\neq$ \\
\hline 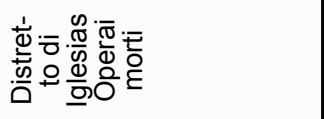 & 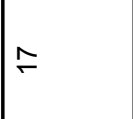 & నิ్త్ర & $\infty$ & $\infty \stackrel{\widehat{F}}{=}$ & $\stackrel{\text { OE }}{=}$ & $\infty \stackrel{\widehat{m}}{=}$ & 을 & $\simeq \widehat{\sigma}$ \\
\hline 言 & 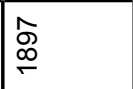 & $\begin{array}{l}\stackrel{\infty}{\infty} \\
\stackrel{\infty}{\leftarrow}\end{array}$ & $\underset{\mathscr{D}}{\mathscr{\infty}}$ & ষ্ণ & চ্口- & סิ & 兽 & 兽 \\
\hline
\end{tabular}




\begin{tabular}{|c|c|c|c|c|c|c|}
\hline 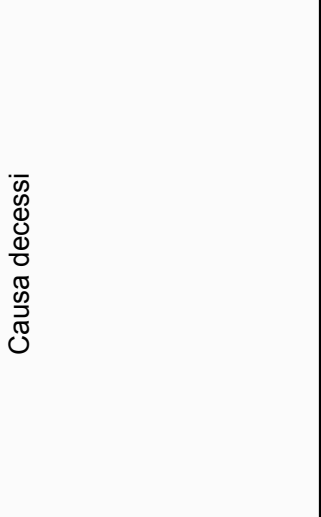 & 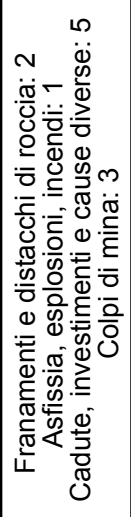 & 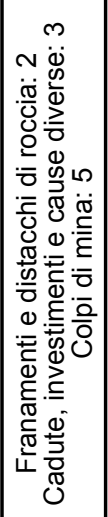 & & 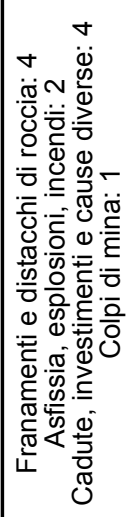 & 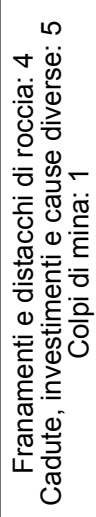 & 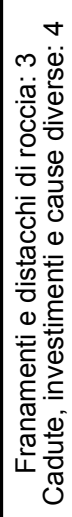 \\
\hline 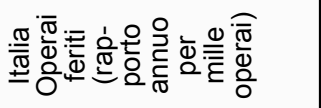 & $\stackrel{\infty}{\stackrel{\infty}{\sim}}$ & $\underset{n}{i}$ & $\frac{\sigma}{m}$ & $\begin{array}{l}\infty \\
\infty \\
-\end{array}$ & $\stackrel{8}{\circ}$ & in \\
\hline 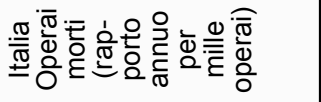 & $\stackrel{\infty}{\stackrel{\infty}{r}}$ & 占 & $\stackrel{8}{\circ}$ & $\stackrel{\text { S }}{\sim}$ & $\stackrel{\bar{m}}{r}$ & 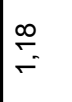 \\
\hline 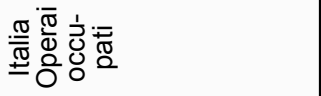 & ஜ & 怘 & 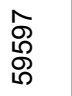 & $\begin{array}{l}- \\
0 \\
0 \\
0 \\
0 \\
10\end{array}$ & 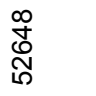 & 迎 \\
\hline 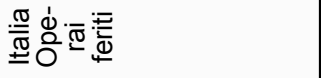 & 욤 & $\underline{10}$ & ㅇ & 응 & 음 & $\stackrel{\sim}{\stackrel{2}{\sim}}$ \\
\hline 焉 & $\stackrel{⿱}{2}$ & à & $\stackrel{m}{\rightleftharpoons}$ & ח & 8 & 只 \\
\hline 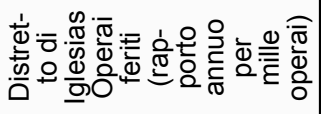 & $\begin{array}{l}\mathscr{2} \\
\infty \\
0\end{array}$ & $\mid \begin{array}{l}\infty \\
\infty \\
0\end{array}$ & $\stackrel{\infty}{m}$ & 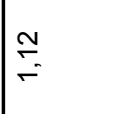 & $\begin{array}{l}1 \\
0 \\
0 \\
0\end{array}$ & $\stackrel{8}{\circ}$ \\
\hline 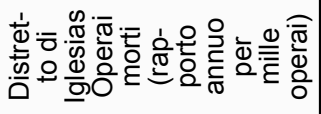 & 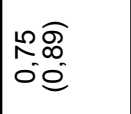 & 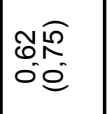 & $\stackrel{\text { N }}{\sim}$ & $\begin{array}{l}\text { No } \\
\hat{0}=0\end{array}$ & $\begin{array}{l}\mathcal{N} \\
\text { No } \\
0=0\end{array}$ & 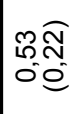 \\
\hline 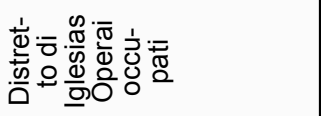 & $\frac{L}{\frac{L}{5}}$ & 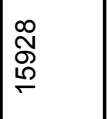 & 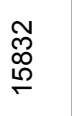 & 음 & $\begin{array}{l}\mathscr{0} \\
\stackrel{\infty}{\infty} \\
\stackrel{m}{\Gamma}\end{array}$ & $\underset{\frac{2}{2}}{\stackrel{2}{2}}$ \\
\hline 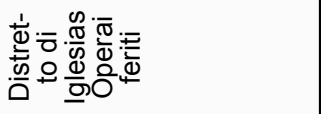 & $\stackrel{m}{\sim}$ & $\stackrel{m}{\square}$ & N & $\approx$ & $\infty$ & $\stackrel{\square}{\square}$ \\
\hline 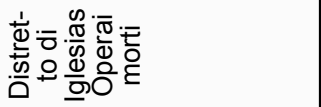 & $\mp \stackrel{\widehat{m}}{=}$ & 은 & TE & $=0$ & 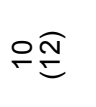 & 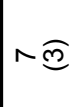 \\
\hline 产 & 욤 & $\begin{array}{l}0 \\
\\
\end{array}$ & 옹 & @ & 움 & 음 \\
\hline
\end{tabular}




\section{Bibliografia:}

Commissione parlamentare d'inchiesta sulla condizione degli operai delle miniere della Sardegna 1910, Atti della Commissione, II, Studi, statistiche e documenti allegati, Roma: Tipografia della Camera dei Deputati.

Commissione parlamentare d'inchiesta sulla condizione degli operai delle miniere della Sardegna 1911, Atti della Commissione, I, Relazione riassuntiva e allegati, Roma: Tipografia della Camera dei Deputati.

«Engineering and Mining Journal», 1910, 10.

Frongia G. 1911, Igiene e miniere in Sardegna, Roma: Tipografia F. Centenari.

Giordano A. 1913, La fisiopatologia e l'igiene dei minatori, Roma: Tipografia nazionale di G. Bertero e C.

Launay de L. 1908, La Conquête Minérale, Paris: Flammarion.

Manconi F. 1986, L'economia e la società delle miniere dall'unità al fascismo, in Le miniere e i minatori della Sardegna, Cagliari: Consiglio regionale della Sardegna, 65-80.

Peis Concas I. 2006, Presenza e lavoro delle donne sarde nelle miniere, in Sardegna: minatori e memorie, Iglesias: Associazione Minatori-Memoria, 41-66.

Pellati N. 1893, Prefazione, "Rivista del Servizio minerario», VII-X.

Porrà R. 1993, Infortuni sul lavoro nelle miniere sarde (1861-1886), in Kirova T. K. (ed.) 1993, L'uomo e le miniere in Sardegna, Cagliari: Edizioni Della Torre, 65-80.

Puddu S., Locci F. 2006, Completamento, riordino e informatizzazione delle ricerche sui minatori caduti, in Sardegna: minatori e memorie, Iglesias: Associazione MinatoriMemoria, 187-292.

Relazione sul servizio minerario nel 1879, 1882, "Annali di Agricoltura».

Ruju S. 1999, I mondi minerari in Sardegna e il caso dell'Argentiera, in Musso S. (ed.) 1999, Tra fabbrica e società. Mondi operai nell'Italia del Novecento, Milano: Feltrinelli, 313-380.

Serra S. 2006, Miniere e minatori di Sardegna. Un'antica civiltà del futuro, in Sardegna: minatori e memorie, Iglesias: Associazione Minatori-Memoria, 75-117.

Todde E. 2013, Sicurezza, infortuni e scioperi nella miniera di Montevecchio nel corso del Novecento, "Bollettino Storico, Archivistico e Consolare del Mediterraneo», 3: 295312.

Vinelli M. 1914, Note sull'industria, la mano d'opera e la legislazione nelle miniere di Sardegna, Cagliari: Società tipografica sarda. 